Article

\title{
Twinning-Detwinning Behavior during Cyclic Deformation of Magnesium Alloy
}

\section{Soo Yeol Lee ${ }^{1, *}$, Huamiao Wang ${ }^{2}$ and Michael A. Gharghouri ${ }^{3}$}

1 Department of Materials Science and Engineering, Chungnam National University, Daejeon 305-764, Korea

2 Materials Science and Technology, Los Alamos National Laboratory, Los Alamos, NM 87544, USA; E-Mail: wanghm@lanl.gov

3 Canadian Neutron Beam Centre, Canadian Nuclear Laboratories, Chalk River, ON K0J 1J0, Canada; E-Mail: Michael.Gharghouri@cnl.ca

* Author to whom correspondence should be addressed; E-Mail: sylee2012@cnu.ac.kr; Tel.: +82-42-821-6637; Fax: +82-42-821-5850.

Academic Editor: Klaus-Dieter Liss

Received: 20 April 2015 / Accepted: 18 May 2015 / Published: 26 May 2015

\begin{abstract}
In situ neutron diffraction has been used to examine the deformation mechanisms of a precipitation-hardened and extruded Mg-8.5wt.\%Al alloy subjected to (i) compression followed by reverse tension (texture T1) and (ii) tension followed by reverse compression (texture T2). Two starting textures are used: (1) as-extruded texture, T1, in which the basal pole of most grains is normal to the extrusion axis and a small portion of grains are oriented with the basal pole parallel to the extrusion axis; (2) a reoriented texture, T2, in which the basal pole of most grains is parallel to the extrusion axis. For texture $\mathrm{T} 1$, the onset of extension twinning corresponds well with the macroscopic elastic-plastic transition during the initial compression stage. The non-linear macroscopic stress/strain behavior during unloading after compression is more significant than during unloading after tension. For texture T2, little detwinning occurs after the initial tension stage, but almost all of the twinned volumes are detwinned during loading in reverse compression.
\end{abstract}

Keywords: magnesium alloy; deformation; twinning; detwinning; neutron diffraction 


\section{Introduction}

Many investigations have been devoted to understanding the deformation behavior of magnesium and its alloys, because of their potential applications for lightweight materials in the aircraft and automotive industries, and for portable electronic devices [1,2]. Magnesium alloys have poor formability at room temperature, which can be attributed to the limited number of available slip systems $[3,4] .<$ a $>$ slip with a $1 / 3<11.0>$ Burgers vector on the close-packed $(00.2)$ basal plane is the primary slip system in hexagonal close-packed (HCP) magnesium. Non-basal $<\mathrm{a}>$ slip on the $\{10.0\}$ prismatic and $\{10.1\}$ pyramidal plane has often been observed at higher stresses. While none of these slip modes can accommodate deformation along the c-axis, deformation twinning can provide limited deformation along the c-axis. $\{10.2\}<10.1>$ extension twinning is commonly found during plastic deformation at room temperature in favorably oriented grains relative to the applied loading direction, leading to tension-compression yield asymmetry and strong plastic anisotropy in magnesium alloys [5-8]. When deformation twinning occurs, the lattice is reoriented approximately $86.3^{\circ}$ relative to the parent lattice, resulting in pronounced changes in the crystallographic texture during deformation [9-12]. In situ neutron diffraction experiments have been employed extensively to study the plastic deformation behavior of magnesium alloys. The technique provides information on the distribution of internal stresses and strains among the various crystallographic orientations, as well as on bulk texture evolution caused by twinning [13-19].

In the current work, neutron diffraction is used to study the plastic deformation behavior of a precipitation-hardened and extruded Mg-8.5wt.\%Al alloy. Lattice strains and diffraction peak intensities for several grain orientations are monitored in situ during deformation to examine the evolution of the stress state and the occurrence of twinning and detwinning in various grain orientations. The loading paths consist of (i) compression followed by reverse tension and (ii) tension followed by reverse compression.

\section{Experimental Section}

\subsection{Materials}

The extruded Mg-8.5wt.\%Al alloy used in this study was solution treated and aged, resulting in equiaxed grains with an average size of $\sim 60 \mu \mathrm{m}$. Details of the sample preparation are provided elsewhere [13]. The bulk crystallographic texture was measured using the E3 neutron diffractometer of the Canadian Neutron Beam Centre, located at the National Research Universal (NRU) reactor at Canadian Nuclear Laboratories, Canada. The orientation distribution function (ODF) for each sample was determined from the $\{10.0\},\{00.2\},\{10.1\}$ and $\{10.2\}$ pole figures. Two starting textures were used: (1) as-extruded texture, T1 (Figure 1a), in which the basal pole of most grains is normal to the extrusion axis and a very small portion of grains are oriented with the basal pole parallel to the extrusion axis; (2) a reoriented texture, T2 (Figure 1b), in which the basal pole of most grains is parallel to the extrusion axis. The reoriented texture, T2, was obtained by compressing the T1 texture along the extrusion direction to a strain of $\sim 9 \%$, followed by annealing at a suitable temperature [18]. Therefore, $\{10.2\}$ extension twinning could be easily activated under compression along the extrusion direction for $\mathrm{T} 1$, and under tension along the extrusion direction for $\mathrm{T} 2$. 

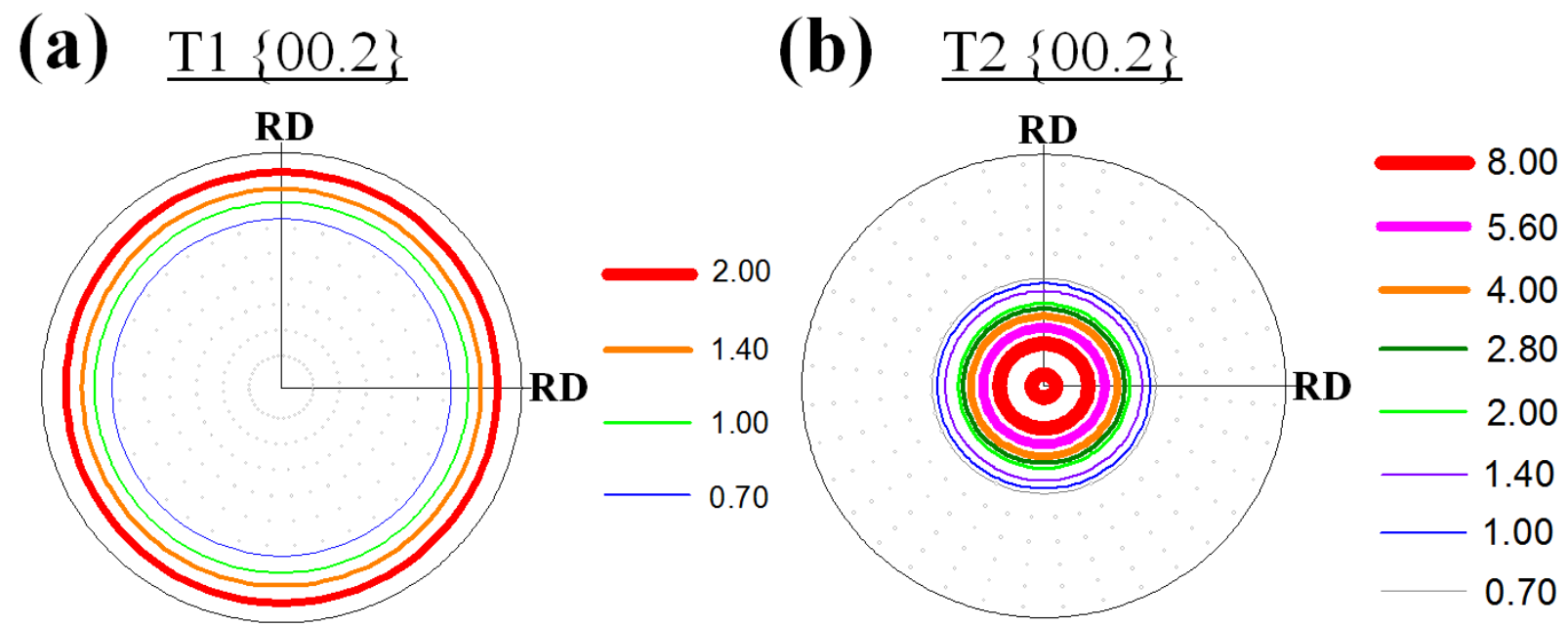

Figure 1. Initial textures of (a) T1 and (b) T2 samples determined by neutron diffraction. The center of each pole figure corresponds to the extrusion direction (ED), RD is the radial direction of the extruded bar.

\subsection{Neutron Diffraction Strain Measurements}

Neutron diffraction strain measurements were conducted on the L3 neutron diffractometer of the Canadian Neutron Beam Centre, Canadian Nuclear Laboratories. T1 and T2 samples were deformed in compression followed by reverse tension and in tension followed by reverse compression, respectively. Diffraction peaks for several grain families, characterized by the crystallographic plane which was normal to the loading direction, were acquired in situ during deformation, yielding interplanar spacings ( $d$-spacings) parallel to the loading direction for each grain family as a function of applied load. The lattice strains for each family were calculated using the following equation:

$$
\varepsilon_{\mathrm{hk} . \mathrm{l}}=\left(d_{h k . l}-d_{h k . l}^{0}\right) / d_{h k . l}^{0}
$$

where $d_{h k . l}^{0}$ and $d_{h k . l}$ are the $d$-spacings of the $\{h k . l\}$ family of planes in the unloaded and loaded conditions, respectively.

\section{Results and Discussion}

The macroscopic stress-strain response of the T1 sample subjected to compression followed by reverse tension is shown in Figure 2.

The material yields at $\sim-100 \mathrm{MPa}$, at which the elastic-plastic transition is well underway, though plasticity is not fully developed. The non-linear macroscopic stress/strain behavior during unloading after compression is more significant than during unloading after tension. 


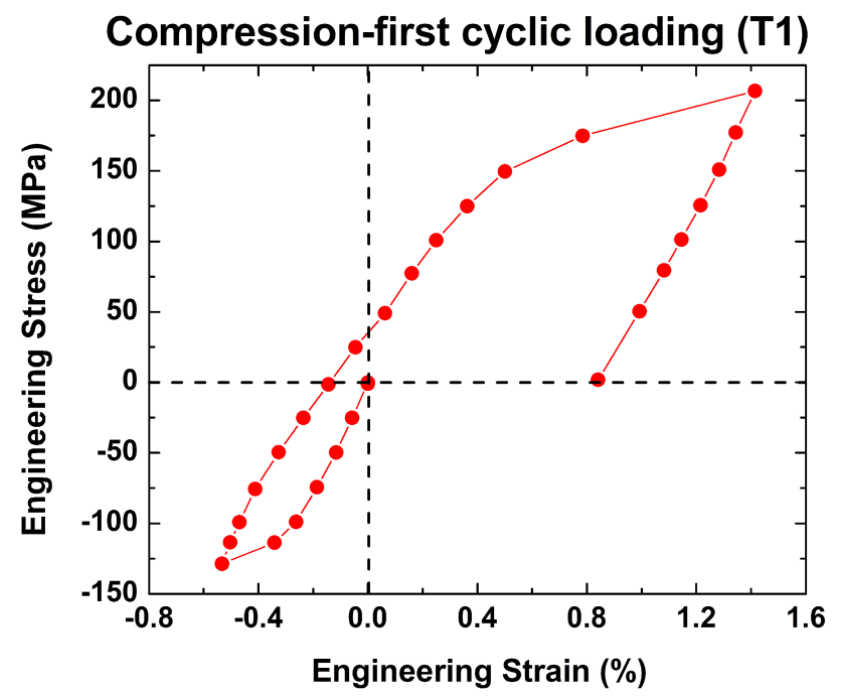

Figure 2. Macroscopic stress-strain response for the T1 sample. The symbols correspond to points in the loading history at which diffraction data were acquired.

Figure 3 shows lattice strain evolution for four different families as a function of applied stress for the T1 sample subjected to compression followed by reverse tension (Figure 2). The corresponding integrated intensity variations for all measured reflections are provided in Figure 4 [17]. A detailed discussion of the intensity variations in Figure 4 is provided in [17], based on which it is possible to identify the stress intervals over which twinning and detwinning occur. The twinning and detwinning intervals identified in Figure 3 are based on this previous analysis.

\section{Compression-first cycling loading (T1)}

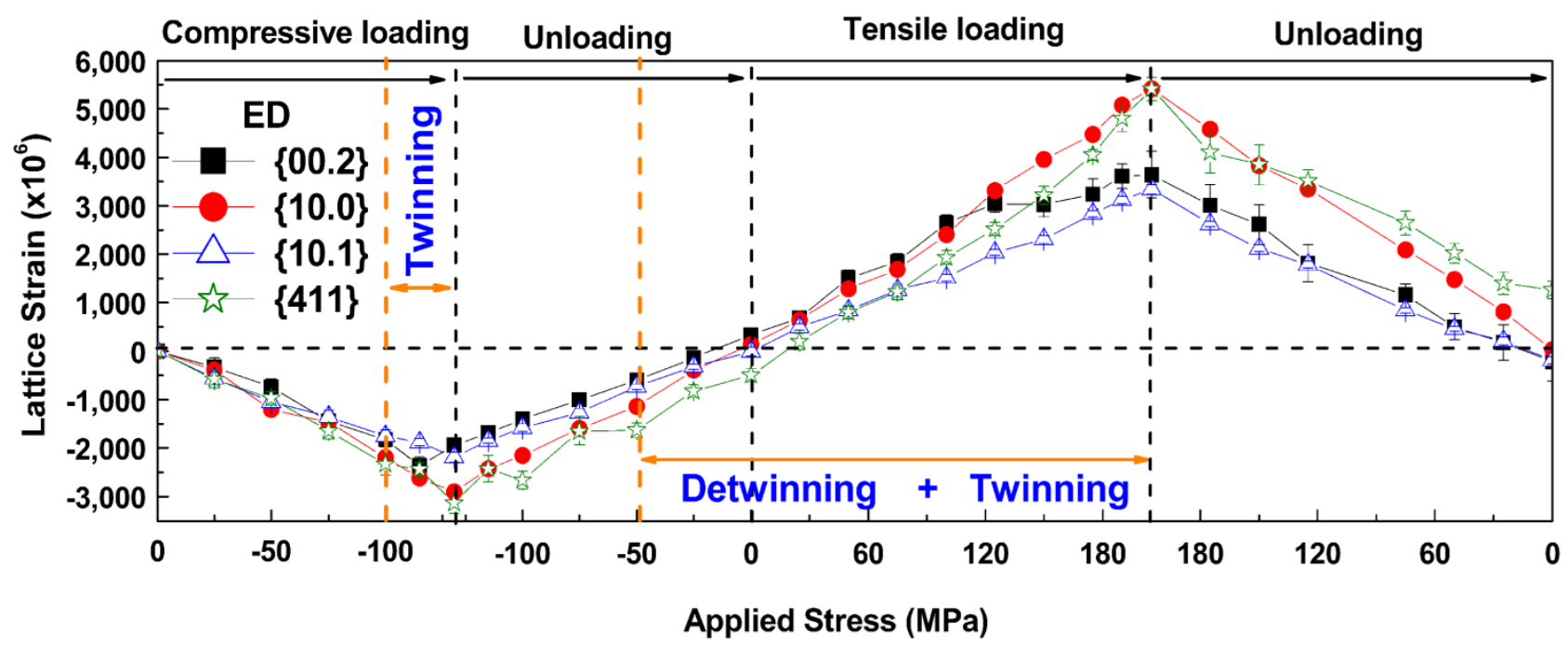

Figure 3. Lattice strain evolution for various grain families as a function of applied stress during the deformation shown in Figure 2. The $\{h k . l\}$ plane normal is parallel to the applied loading direction, which is parallel to the extrusion direction (ED). The angles between the c-axis and the loading direction for the $\{00.2\},\{10.0\}$ and $\{10.1\}$ families are $0^{\circ}, 90^{\circ}$ and $61.9^{\circ}$, respectively. 


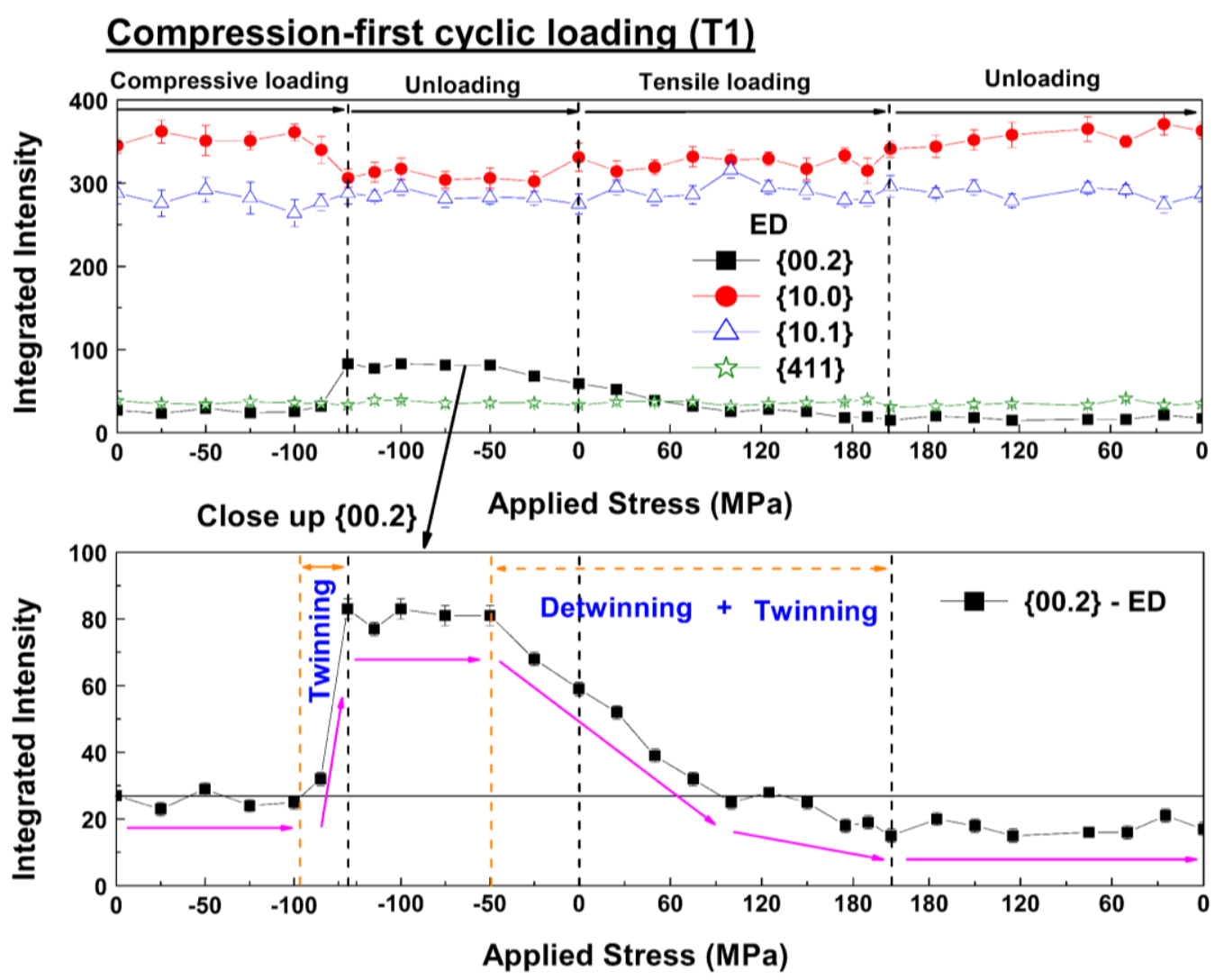

Figure 4. Intensity variations for various grain families as a function of applied stress during the deformation shown in Figure 2 [17].

In Figure 3, during the initial compressive loading step, the lattice strain increases linearly with applied stress for all four grain families up to $\sim-50 \mathrm{MPa}$. Beyond this stress, the lattice strain in the $\{10.1\}$ family increases more slowly with applied stress compared with the other orientations, indicating that this family deforms plastically. The deformation mechanism is most likely basal $<\mathrm{a}>$ slip, which has the lowest critical resolved shear stress (CRSS) of all the commonly observed deformation modes, and for which the $\{10.1\}$ family is favorably oriented. As the load increases beyond $\sim-100 \mathrm{MPa}$, the $\{00.2\}$ and $\{10.0\}$ lattice strains deviate from one another, corresponding to an increase in the $\{00.2\}$ intensity and a concurrent decrease in the $\{10.0\}$ intensity (see Figure 4 [17]). Despite being favorably oriented for $\{10.2\}<10.1>$ extension twinning in compression, the strain in the $\{10.0\}$ family increases linearly with the applied stress during the initial compressive loading step, resulting in almost zero residual strain upon unloading. On the other hand, the lattice strain in the $\{00.2\}$ family, which is twin-related to the $\{10.0\}$ grains, experiences relaxation during the final loading step, resulting in a slightly tensile residual strain at the end of unloading. The residual strain for the soft $\{10.1\}$ family is slightly compressive, or zero. The strain in the $\beta-\mathrm{Mg}_{17} \mathrm{Al}_{12}$ precipitates ( $\{411\}$ reflection) increase linearly during loading, with no evidence of relaxation, resulting in relatively large compressive residual strains upon unloading. During unloading after compression, it was found that about $40 \%$ of the twinned volume detwins [17]. This detwinning behavior is thought to contribute significantly to the non-linear behavior observed in Figure 2.

During reverse loading in tension, the intensity data in Figure 4 [17] show that detwinning continues up to an applied stress of $\sim+100 \mathrm{MPa}$, after which the $\{00.2\}$ minority grains undergo 
$\{10.2\}$ extension twinning, as revealed by a decrease in the $\{00.2\}$ intensity beyond $\sim 100 \mathrm{MPa}$. The $\{00.2\}$ and $\{10.1\}$ families, favorably oriented for extension twinning and basal slip respectively, show stress relaxation during reverse tensile loading, resulting in slightly compressive residual strains upon unloading. Conversely, the lattice strain in the $\{100\}$ family, which is not favorably oriented for basal slip or extension twinning, varies linearly throughout the test, resulting in negligible residual strain at the end of the test. The $\{411\}$ precipitates accumulate high levels of lattice strain during the reverse tensile loading, but, unlike the $\{10.0\}$ family, the precipitates show large tensile residual strains upon final unloading.

Figure 5 shows the macroscopic stress-strain response for the T2 sample subjected to tension followed by reverse compression. The initial stress-strain response is linear up to an applied stress of $\sim 75 \mathrm{MPa}$, after which the slope decreases as the material starts to yield. During unloading after tension and reloading in compression, the stress-strain response is clearly non-linear. The material undergoes general yielding in compression at $\sim-50 \mathrm{MPa}$. The unloading behavior at the end of the test is also clearly non-linear, but the effect is less significant than the non-linear behavior after compression for T1 (Figure 2).

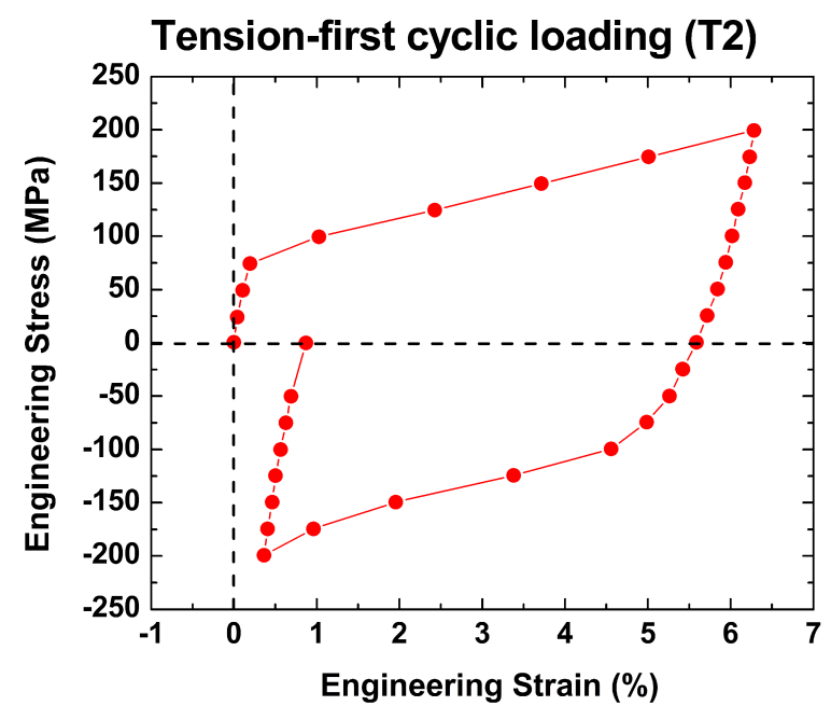

Figure 5. Macroscopic stress-strain response for the T2 sample. The symbols correspond to points in the loading history at which diffraction data were acquired.

Lattice strain and integrated intensity variations for four different grain orientations as a function of applied stress are shown in Figure 6 for the T2 sample subjected to tension followed by reverse compression.

During initial loading in tension, the lattice strain varies linearly with applied stress for all four grain orientations up to $\sim 50 \mathrm{MPa}$ showing the same slope, as expected based on the near elastic isotropy of magnesium. Beyond $\sim 50 \mathrm{MPa}$, the $\{10.2\}$ and $\{10.3\}$ strains increase more slowly with stress, indicating that these grains undergo plastic deformation, most likely by basal $<a>$ slip (Figure 6a), which is the easiest slip system to activate in $\mathrm{Mg}-\mathrm{Al}$ alloys, and for which these grain families are favorably oriented. Beyond $\sim 75 \mathrm{MPa}$, the lattice strain behavior of the $\{00.2\}$ family deviates from its initial linear behavior, corresponding to a decrease in intensity for the $\{00.2\}$ peak in Figure 6b, and macroscopic yielding in Figure 5. The $\{00.2\}$ family, which represents a large fraction 
of the microstructure, has the basal pole aligned $0^{\circ}$ relative to the applied loading direction. It is thus favorably oriented for $\{10.2\}$ extension twinning in tension, which results in the observed decrease in intensity. Likewise, the changes in the $\{11.0\}$ and $\{10.3\}$ intensities for $75<\sigma(\mathrm{MPa})<200$ are due to $\{10.2\}$ extension twinning in the $\{10.3\}$ families, as the two families are twin-related.

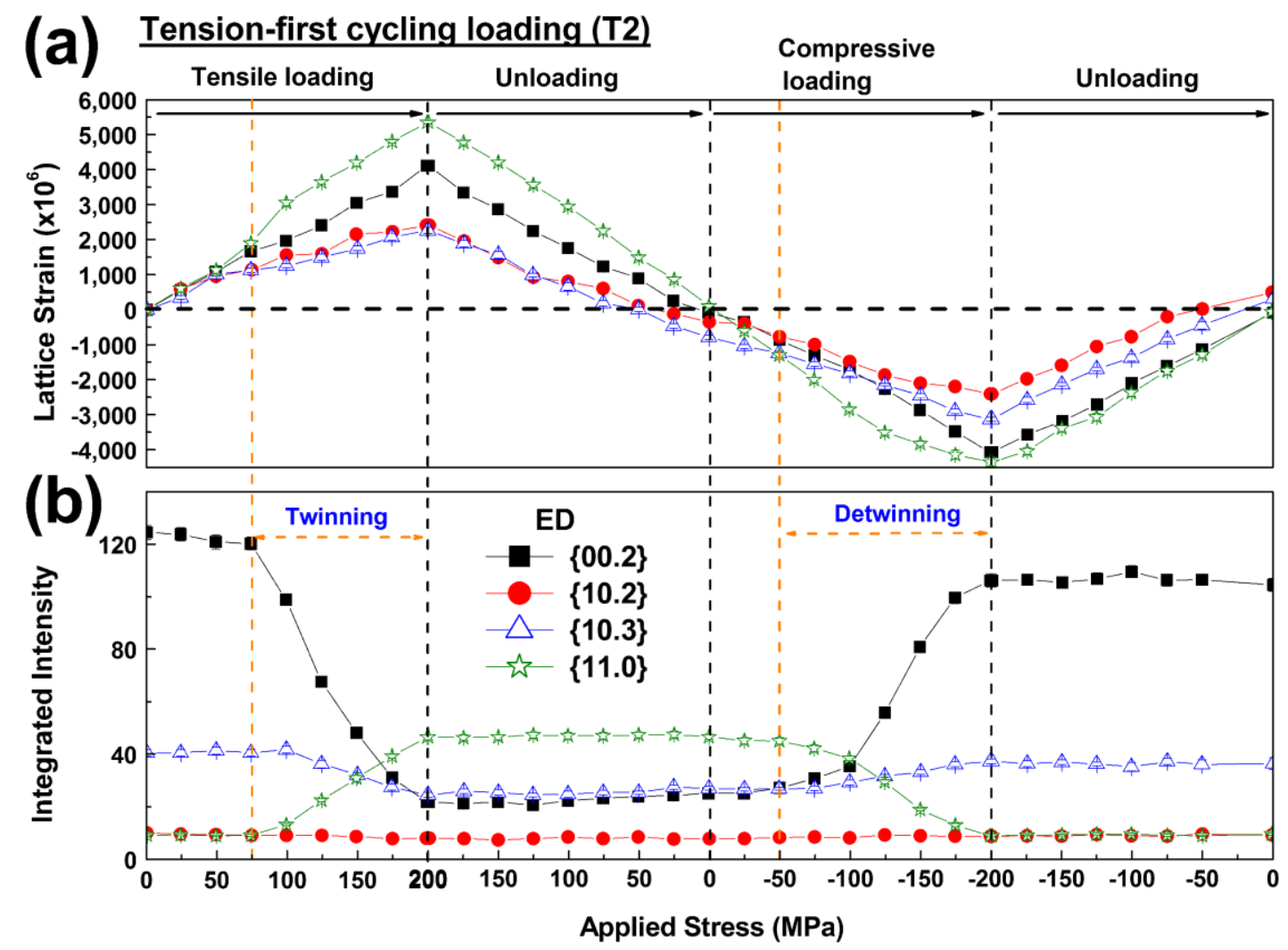

Figure 6. (a) Lattice strain evolution and (b) intensity variations for various grain families as functions of applied stress during the deformation shown in Figure 5. The $\{h k . l\}$ plane normal is parallel to the applied loading direction, which is parallel to the extrusion direction (ED).

Figure $6 \mathrm{a}$ shows that the $\{10.2\},\{10.3\}$ and $\{00.2\}$ families, which deform plastically by either basal slip or extension twinning during the initial tensile portion of the test, undergo stress relaxation, resulting in compressive residual strains upon unloading. On the other hand, the $\{11.0\}$ family, which is unfavorably oriented for both extension twinning and basal slip, accumulates much higher lattice strains during tensile loading, resulting in slightly tensile residual strains at the end of unloading. In the compression stage of the test, the $\{10.2\}$ and $\{10.3\}$ families, which are favorably oriented for basal slip, accumulate relatively low levels of lattice strain during loading, resulting in small tensile residual strains at the end of the test. Conversely, the residual strain in the hard $\{00.2\}$ family is slightly compressive, or zero. The $\{11.0\}$ family presents an interesting case-despite being favorably oriented for extension twinning in compression, it still accumulates large lattice strains during loading, resulting in slightly negative (or zero) residual lattice strain at the end of the test. This lack of a significant relaxation effect associated with twinning is consistent with the behavior of the $\{10.0\}$ family during the initial compressive loading step in T1, and is in marked contrast to the behavior of the $\{00.2\}$ family in both textures, in which strong relaxation effects are associated with twinning. This difference 
in behavior between the $\{10.0\} /\{11.0\}$ and $\{00.2\}$ orientations may be due to the fact that extension twinning is activated by compression normal to the c-axis in the $\{10.0\}$ and $\{11.0\}$ family, but by tension parallel to the c-axis in the $\{00.2\}$ family.

During unloading after tension, the intensities of all four reflections change little (the $\{00.2\}$ and $\{10.3\}$ intensities increase slightly), indicating that the twinned material generated during tension does not undergo significant detwinning. During reverse loading in compression, however, the twinned volume within the $\{00.2\}$ family, which contributes to the $\{10.0\}$ signal, is favorably oriented to detwin in compression. Thus, in reverse compression, detwinning starts at an applied stress of $\sim-50 \mathrm{MPa}$ and continues up to an applied stress of $-200 \mathrm{MPa}$, as revealed by the increase in the $\{00.2\}$ intensity (Figure 6b). However, the $\{00.2\}$ intensity at the start of the test is not fully recovered at $-200 \mathrm{MPa}$. During unloading after the compressive portion of the test, the $\{00.2\}$ peak intensity does not change, suggesting that the non-linear behavior observed in Figure 5 is not related to the behavior of deformation twins, but rather to dislocation phenomena. This behavior is likely due to the fact that almost all traces of twinning are gone when the highest compressive stress is reached, such that little material is favorably oriented for either twinning or detwinning when the final unloading starts.

\section{Conclusions}

In situ neutron diffraction has been used to investigate the plastic deformation behavior of Mg-8.5wt.\%Al alloy subjected to compression followed by reverse tension (texture T1) and tension followed by reverse compression (texture T2). For texture T1, the onset of extension twinning corresponds well with the macroscopic elastic-plastic transition during the initial compression stage. The non-linear macroscopic stress/strain behavior during unloading after compression is more significant than during unloading after tension. For texture T2, little detwinning occurs after the initial tension stage, but almost all of the twinned volumes are recovered during loading in reverse compression. The development of residual strains in the various grain orientations during unloading after compression is strongly influenced by plastic anisotropy at the grain level. The residual strains observed in each grain family can be largely rationalized based on the ease of activation of basal slip and extension twinning.

\section{Acknowledgments}

This work was supported by the National Research Foundation of Korea (NRF) grant funded by the Korean government (MSIP) (No.2013R1A4A1069528, No.2013R1A1A1076023).

\section{Author Contributions}

S.Y.L. wrote the initial draft of the manuscript; S.Y.L., H.W., M.A.G. analyzed the data; All coauthors contributed to the interpretation of the data.

\section{Conflicts of Interest}

The authors declare no conflict of interest. 


\section{References}

1. Avedesian, M.M.; Baker, H. Magnesium and Magnesium Alloys; ASM Specialty Handbook; ASM International: Materials Park, OH, USA, 1999.

2. Wang, C.; Han, P.; Zhang, L.; Zhang, C.; Yan, X.; Xu, B.S. The strengthening effect of Al atoms into Mg-Al alloy: A first-principles study. J. Alloy. Comp. 2009, 482, 540-543.

3. Roberts, C.S. Magnesium and Its Alloys; John Wiley \& Sons, Inc.: New York, NY, USA, 1960.

4. Muránsky, O.; Carr, D.G.; Barnett, M.R.; Oliver, E.C.; Šittner, P. Investigation of deformation mechanisms involved in the plasticity of AZ31 Mg alloy: In situ neutron diffraction and EPSC modelling. Mater. Sci. Eng. A 2008, 496, 14-24.

5. Partridge, P.G. The crystallography and deformation modes of hexagonal close-packed metals. Metall. Rev. 1967, 12, 169-194.

6. Agnew, S.R.; Tomé, C.N.; Brown, D.W.; Holden, T.M.; Vogel, S.C. Study of slip mechanisms in a magnesium alloy by neutron diffraction and modeling. Scripta Mater. 2003, 48, 1003-1008.

7. Jain, J.; Poole, W.J.; Sinclair, C.W.; Gharghouri, M.A. Reducing the tension-compression yield asymmetry in a Mg-8Al-0.5Zn alloy via precipitation. Scripta Mater. 2010, 62, 301-304.

8. Hong, S.G.; Park, S.H.; Lee, C.S. Role of $\{10-12\}$ twinning characteristics in the deformation behavior of a polycrystalline magnesium alloy. Acta Mater. 2010, 58, 5873-5885.

9. Clausen, B.; Tomé, C.N.; Brown, D.W.; Agnew, S.R. Reorientation and stress relaxation due to twinning: Modeling and experimental characterization for Mg. Acta Mater. 2008, 56, 2456-2468.

10. Proust, G.; Tomé, C.N.; Jain, A.; Agnew, S.R. Modeling the effect of twinning and detwinning during strain-path changes of magnesium alloy AZ31. Int. J. Plasticity 2009, 25, 861-880.

11. Muránsky, O.; Barnett, M.R.; Luzin, V.; Vogel, S. On the correlation between deformation twinning and Luders-like deformation in an extruded Mg alloy: In situ neutron diffraction and EPSC.4 modelling. Mater. Sci. Eng. A 2008, 527, 1383-1394.

12. Park, S.H.; Hong, S.G.; Lee, C.S. In-plane anisotropic deformation behavior of rolled Mg-3Al-1Zn alloy by initial $\{10-12\}$ twins. Mater. Sci. Eng. A 2013, 570, 149-163.

13. Gharghouri, M.A.; Weatherly, G.C.; Embury, J.D.; Root, J. Study of the mechanical properties of Mg-7.7at.\% Al by in situ neutron diffraction. Phil. Mag. A 1999, 79, 1671-1695.

14. Brown, D.W.; Agnew, S.R.; Bourke, M.A.M.; Holden, T.M.; Vogel, S.C.; Tomé, C.N. Internal strain and texture evolution during deformation twinning in magnesium. Mater. Sci. Eng. A 2005, 399, 1-12.

15. Wu, L.; Agnew, S.R.; Brown, D.W.; Stoica, G.M.; Clausen, B.; Jain, A.; Fielden, D.E.; Liaw, P.K. Internal stress relaxation and load redistribution during the twinning-detwinning-dominated cyclic deformation of a wrought magnesium alloy, ZK60A. Acta Mater. 2008, 56, 3699-3707.

16. Muránsky, O.; Barnett, M.R.; Carr, D.G.; Vogel, S.C.; Oliver, E.C. Investigation of deformation twinning in a fine-grained and coarse-grained ZM20 Mg alloy: Combined in situ neutron diffraction and acoustic emission. Acta Mater. 2010, 58, 1503-1517.

17. Lee, S.Y.; Gharghouri, M.A.; Root, J.H. Plastic deformation of magnesium alloy subjected to compression-first cyclic loading. In Proceeding of the 140th TMS Annual Meeting \& Exhibition, San Diego, CA, USA, 27 February-3 March 2011; pp. 595-598. 
18. Lee, S.Y.; Gharghouri, M.A. Pseudoelastic behavior of magnesium alloy during twinning-dominated cyclic deformation. Mater. Sci. Eng. A 2013, 572, 98-102.

19. Lee, S.Y.; Wang, H.; Gharghouri, M.A.; Nayyeri, G.; Woo, W.; Shin, E.; Wu, P.D.; Poole, W.J.; Wu, W.; An, K. Deformation behavior of solid-solution-strengthened Mg-9 wt.\% Al alloy: In situ neutron diffraction and elastic-viscoplastic self-consistent modeling. Acta Mater. 2014, 73, 139-148.

(C) 2015 by the authors; licensee MDPI, Basel, Switzerland. This article is an open access article distributed under the terms and conditions of the Creative Commons Attribution license (http://creativecommons.org/licenses/by/4.0/). 